\title{
FOOTPRINTS OF AN ACTIVE GALACTIC NUCLEUS: THE NUCLEAR ZONE OF NGC 1068
}

\author{
BRUCE BALICK ${ }^{\text {a), b) }}$ \\ Sterrewacht, Leiden University, Leiden, The Netherlands and University of Washington, Department of Astronomy, \\ FM-20, Seattle, Washington 98195 \\ Timothy HeCKMAN ${ }^{a), c)}$ \\ Astronomy Program, University of Maryland, College Park, Maryland 20742 \\ Received 28 June 1984; revised 12 October 1984
}

\begin{abstract}
NGC 1068 is a spiral galaxy that hosts an extremely luminous active nucleus imbedded in an unusually bright gaseous medium. The galaxy is nearby and, unlike Cen A, its near-nuclear region is plainly visible. Therefore the galaxy presents an exceptionally propitious opportunity to separate clues to the origin of nuclear activity from the disruptions resulting from this activity. The many observational data, including new, deep emission-line images and long-slit spectra, are reviewed with this aim. Though no firm conclusions emerge, a variety of important new and potentially viable research directions are identified. NGC 1068 appears to be a hybrid Seyfert/starburst galaxy.
\end{abstract}

\section{INTRODUCTION}

Since the discovery of active galactic nuclei, observational and theoretical work in the field has been paced by questions of nuclear phenomenology and the nature of the "machine" or "monster" that generates this nuclear energy. Two complementary questions are: (How) does the nuclear environment affect nuclear activity (see review by Balick and Heckman 1982)? and the reverse: (How) does nuclear activity affect the environment? The first question is one of cause: Is nuclear activity triggered and sustained by external "fuel"? The second question is one of effect: Does nuclear activity disrupt the host galaxy and the adjacent extragalactic environment? Here we examine both questions.

In the present paper, attention is turned to the active galaxy NGC 1068 and its near-nuclear regions. Scientifically, the galaxy is of special interest owing to its extremely large luminosity among Seyfert galaxies (Telesco and Harper 1980 -hereafter referred to as TH) and its relatively gas-rich nuclear surroundings (e.g., Walker 1968) in which clues to the origin or impact of nuclear activity might be inscribed. Operationally, NGC 1068 offers the advantages of proximity (i.e., good spatial scale), inclination (unlike Cen A, its nuclear zone is clearly exposed), and familiarity (NGC 1068 has been studied using an impressive array of modern techniques). Moreover, it is visible from both terrestrial hemispheres.

The original purpose of the paper was to review the key observations and interpretations of NGC 1068. However, the paper has developed an extra purpose of its own volition. The synthesis of earlier work plus some unpublished emission-line images leads us to point out some important new observations already viable from the ground and other easily possible observations in space. Therefore we offer the work as a "review and preview" article.

Limitations of space and stylistic coherence drive us to emphasize certain aspects of the literature concerning NGC 1068. No pretense to completeness is offered. We hope that most of our omissions are thoughtful and intentional.

\section{PAST OBSERVATIONS}

Burbidge, Burbidge, and Pendergast (1959), Bertola (1968), and Walker (1968) first pointed out many of the interesting features within about $20^{\prime \prime}\left(\sim 1 \mathrm{kpc}^{*}\right)$ of the nucleus of NGC 1068 that are unusual for a galaxy of the same (Sb) Hubble type. For example, Bertola's pictures in blue light showed large numbers of bright star clusters within a $30^{\prime \prime} \times 20^{\prime \prime}$ elliptical region that we define as the near-nuclear zone (NZ). Walker found that gas motions within $\sim 15^{\prime \prime}$ of the nucleus showed strong radial components ranging from -600 to $+600 \mathrm{kms}^{-1}$. This high-velocity gas was observed in the $\mathrm{O}$ III line and its spatial distribution was found to be dissimilar from that of $\mathrm{H}$ II regions near the nucleus (Alloin et al. 1981) or other known morphological features discussed below. Walker identified four high-velocity cloudlike features (kinematic "clouds") extending $\sim 7$ " from the nucleus in several directions.

Since Walker's work, a variety of spectroscopic observations (Alloin et al. 1983-hereafter referred to as A83; Scoville, Young, and Lucy 1983-hereafter referred to as SYL83; Pelat and Alloin 1980 and references therein; Balick and Heckman 1979-hereafter referred to as BH79) have broadly confirmed the presence of these radial motions deep in the NZ. In addition, ultraviolet observations by Snijders, Briggs, and Boksenberg (1982-hereafter referred to as SBB82) have shown that the UV luminosities and ionization properties of some of the giant $H$ II regions at the edge of the NZ are comparable to those of the nucleus itself. SBB82 and Weedman and Huenemoerder (1984) suggest that a burst of massive star formation, similar to that in NGC 7714 (Weedman et al. 1981), is being observed. Mapping of the molecular gas $(\mathrm{CO})$ and warm dust in the NZ (SYL83 and Telesco et al. 1984 -hereafter referred to as TBWH) has provided additional evidence for a "starburst" on a scale of several kpc in NGC 1068. Such a burst may have been initiated by the impact of nuclear activity, as modeled by Sanders and Bania (1976). However, recent observations by Nishimura et al. (1984-hereafter referred to as N84) seem to suggest that

\footnotetext{
a) Guest observers at National Optical Astronomy Observatory (NOAO) NOAO is operated by Associated Universities for Research in Astronomy, Inc., under contract with the National Science Foundation.

b) Written while on leave from the University of Washington, Seattle, WA.

c) Alfred P. Sloan Foundation Fellow.
}

*All distance-dependent parameters described in this paper are referred to $H_{0}=100 \mathrm{kms}^{-1} \mathrm{Mpc}^{-1}$. We abbreviate north, east, south, and west as $\mathrm{N}$, E, S, and W, respectively. [O III] 5007 will be designated $\mathrm{O}$ III, and $\mathrm{H} \alpha 6563$ plus $[\mathrm{N} \mathrm{II]} 6548+6583$ will be designated $(\mathrm{H}+\mathrm{N}$ II). 
sources of heating and ionization other than hot stars can be important as far as 15-30" NE from the nucleus.

The only other spectral region for which detailed maps of the NZ are available is the radio. Wilson and Ulvestad (1982-hereafter referred to as WU82; 1983-hereafter WU83), Van der Hulst, Hummel, and Dickey (1982) and Pedlar et al. (1983), among others, have mapped the radio continuum at a variety of frequencies and spatial resolutions. The radio surface brightness of the disk is generally very high (WU82), implying a global disturbance of some sort. Within 5 " of the nucleus are two prominent, compact, and complex "jets" on opposite quadrants of the nucleus (WU83). Each of the two jet lobes has the luminosity of $\sim 10^{4}$ Cas A SNRs. The polarization, spectral index, and structure of the jets argue that the radio emission does not arise from a young massive population of evolving stars (WU82). Rather, the radio jets seem to have properties shared with the lobes and jets of radio galaxies, and may be formed and shaped in exactly the same way (Icke 1984).

The NZ is not only rich in phenomena, but among Seyfert galaxies which have been systematically imaged by us in the light of $\mathrm{O}$ III and $\mathrm{H}+\mathrm{N}$ II, it shows the greatest number of peculiarities (the images of NGC 1068 are presented below). The large number of identified features is presumably, though not necessarily, related to both the proximity of NGC 1068 and to the very great level of nuclear activity as implied by the large luminosity of the nucleus, e.g., TH measure $\sim 10^{11.5} L_{\odot}$ in the infrared alone. Also plentiful near the nucleus is gas $\left(\sim 10^{9} M_{\odot}\right.$ in the molecular form alone; SYL83) and dust $\left(\sim 10^{7} M_{\odot} ; \mathrm{TH}\right)$. The near-nucleus gas in particular can serve both as a source of fuel for nuclear activity and as an "ether" in which the impact of this activity may be viewed. That some form of "communication" occurs between the nucleus and points in the disk $\geqslant 1 \mathrm{kpc}$ distant is clear from the results of Bertola, Walker, BH79, A83, Hildebrand et al. (1977), and many others.

\section{NEW OBSERVATIONS}

The relationships which may exist between the active nucleus of NGC 1068 and the numerous giant $\mathrm{H}$ II regions, kinematic clouds, radio jets, molecular clouds, etc., nearby are probably best probed through imaging. The most direct approach is through images taken in the light of gaseous emission lines at optical wavelengths, e.g., $\mathrm{O}$ III, $(\mathrm{H}+\mathrm{N}$ II), etc. A few such images are available in the literature (e.g., Bertola 1966; Alloin et al. 1981), but all of these include starlight as well as emission lines. Therefore we present hitherto unpublished emission-line images in which continuum light has been subtracted. As shown below, a number of subtle but surprising features are revealed in this manner.

The observations presented here consist of optical images of NGC 1068 which were obtained during runs described previously (Heckman and Balick 1979; Balick and Heckman 1981; Heckman et al. 1982). In summary, a SIT vidicon, called a "video camera", was used in conjunction with several 50-70 A-wide filters on the 2.1- and 4-m telescopes of Kitt Peak National Observatory under conditions of excellent seeing and transparency. Frames were obtained through filters that transmit the $\mathrm{O}$ III and the $(\mathrm{H}+\mathrm{N}$ II) lines, respectively. Other frames were made in the continuum $\sim 100 \AA$ to the red of each of the emission lines. The images were processed and combined in order to produce continuum-free images in the lines and line-free images in the continuum. The nucleus saturated the detector in the $2.1-\mathrm{m}$ observations
(1.5 seeing). A suitable neutral-density filter was employed to prevent nuclear saturation in a later 4-m observation (1."2 seeing).

The final results of the $2.1-\mathrm{m}$ observations are shown in Fig. 1. The grey levels (and selected contours) selected for the left-hand panels are intended to emphasize the subtle features; the level spacing is logarithmic. The emission-line frames were convolved with a circular Gaussian $(\mathrm{FWHM}=3$ pixels $=1.25)$ in order to reduce uncorrelated noise, and are redisplayed in the upper two right-hand panels using a more conventional selection of contours and grey levels. The interesting emission-line features of the $\mathrm{NZ}$ are best seen, and the most outstanding ones identified, in the right-hand panels.

The absolute ratio of $\mathrm{O}$ III to $(\mathrm{H}+\mathrm{N}$ II $)$ intensities in these images can not be reliably measured from the present data alone. In earlier publications, it was found that the sensitivity of the video camera with the filters used here is between two and four times greater at about $5000 \AA$ than at $6600 \AA$. In any case, variations in the $\mathrm{O}$ III to $(\mathrm{H}+\mathrm{N}$ II) ratio are reliably discernable except where the video camera operated near saturation.

The $\mathrm{O}$ III frame obtained at the 4-m telescope is shown in Fig. 2 (note the difference in spatial scale relative to Fig. 1). This image confirms the bright $O$ III structures seen in Fig. 1. Also shown in Fig. 2 is the VLA radio image at $\lambda 6 \mathrm{~cm}$ from WU83 reproduced at the same spatial scale as the $O \mathrm{III}$ image.

We have also obtained long-slit spectroscopic data on the NZ using the High Gain Video Spectrometer (HGVS) on the 4-m telescope at KPNO during a run in December 1978 [see Heckman et al. (1982) for details concerning data acquisition and reduction]. The HGVS was configured so as to provide a spectral resolution of $\sim 16 \AA$, and a spatial resolution of $\sim 2-3$ " (2" slitwidth). The effective spectral coverage was from $\lambda 3400 \AA$ to $\lambda 5500 \AA$ and the slit length ws $2.7^{\prime}$. Spectra were acquired along three position angles passing through the nucleus $\left(\mathrm{PA}=40^{\circ}, 60^{\circ}\right.$, and $\left.140^{\circ}\right)$. To protect the HGVS image tube from damage, a "pointer" was inserted in front of the spectrograph slit jaws to block out the central few arcsec of the very bright nucleus. As a result of this procedure, the data within $\sim 8^{\prime \prime}$ of the nucleus are unreliable (the pointer was not exactly in focus, and scattered nuclear light dominates within this inner region). These data are not well calibrated in a relative spectrophotometric sense, but are useful to check the imaging data and to provide information on changes in emission-line ratios (e.g., [O II] $\lambda 3727 /[\mathrm{O}$ III ] $\lambda$ 5007) along the slit.

\section{DISCUSSION}

In this section, we discuss the major structural features found in the NZ other than the unresolved nucleus itself. Not only the identifications of, but also the links between, the many features are described. The discussion proceeds from small to large size scales outside the nucleus.

\section{a) The Inner 5" Region}

Within this radius lie the previously reported radio lobes (e.g., WU83) and high-velocity optical emission-line clouds (Walker 1968, A83, etc.). The possible physical nature of this region has been explored by Jones et al. (1977), Miller and Antonucci (1983), Pedlar et al. (1983), and WU83. On the basis of polarization measurements, Miller and Antonucci have suggested that the nucleus of NGC 1068 is surrounded 

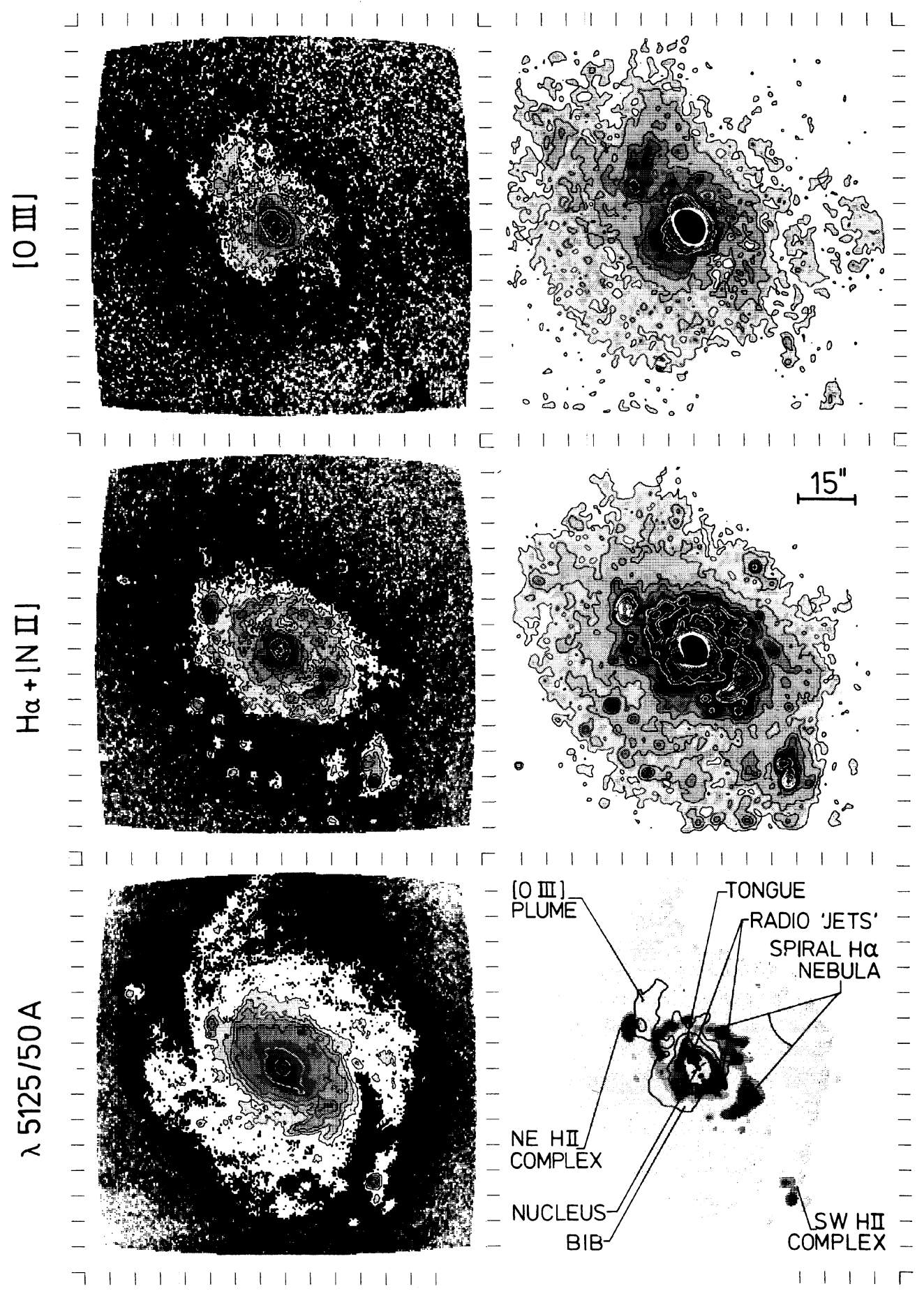

FIG. 1. Images of NGC 1068 in the light of [O III] (top row), $\mathrm{H} \alpha+[\mathrm{N} \mathrm{II}]$ (middle row), and the continuum at $5125 \AA$ A through a 50-A -wide filter (bottom left). Starlight has been subtracted, and the nucleus saturated the detector in the emission-line frames. Absolute brightness levels are unknown. The ratio of system gains at 5025, 5125, and $6600 \AA \AA$ is 1:1:0.4 (approx.). The pixel size is 0.43 , and tic marks are drawn every 16 pixels.

Left panels: Logarithmic representation of the images. Grey scale is used twice. Grey scale changes and selected contours are drawn relative to background as follows: upper and middle frames, $-5,0,5,10,15,20,25,40,63 \ldots(1 / 2 \mathrm{mag}) \ldots 1000$; lower frame, $-10,-5,0,5,10,16,25,40 \ldots(1 / 2 \mathrm{mag}) \ldots 1000$.

Right panels: Top and middle frames are quasilinear representations of [O III] and $(\mathrm{H}+\mathrm{N}$ II) frames after smoothing by a circular Gaussian function, FWHM $=3$ pixels. Grey scale changes and contour levels are drawn at levels 25, 50, 100, 150, $200,300,400,600 \ldots 1600$ with respect to the background level. The lower frame is used to identify important features. Light grey: low-level continuum emission; dark grey: regions of relatively bright $\mathrm{H} \alpha+[\mathrm{N} \mathrm{II}]$ emission outside the nucleus; black: schematic representation of radio lobes (WU83); contours: selected [O III] brightness isophotes. 


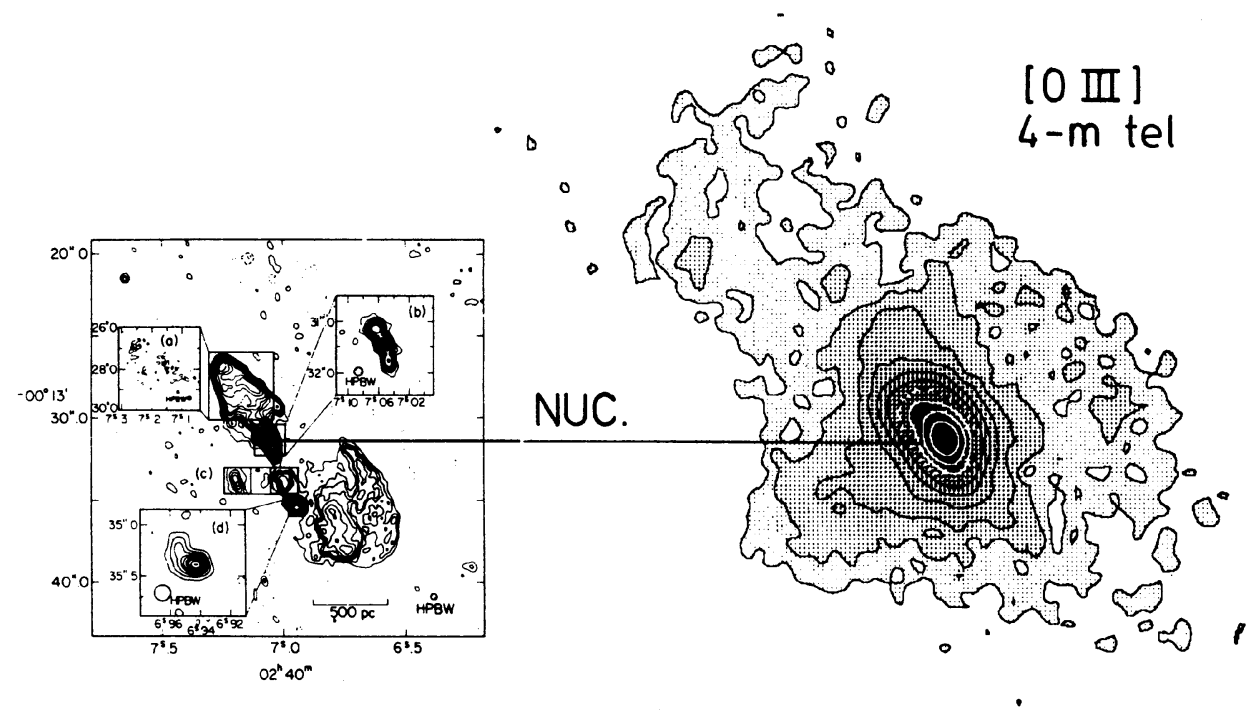

FIG. 2. Image of NGC 1068 in the light of [O III] taken through a neutral-density filter at the 4-m telescope. Starlight has been subtracted. Pixel spacing is 0 "29. Grey scale changes and selected contours are shown at levels of $-10,-5,0,5,10$, $16,25,40 \ldots(1 / 2 \mathrm{mag}) \ldots 25119$ relative to background. The nucleus is not saturated. To the right, the radio brightness isophotes of WU83 are reproduced to the same scale as the [O III] frame shown (refer to WU83 for a better rendering of the radio isophotes).

by an accretion disk whose axis of symmetry would coincide with the radio-jet axis. Though highly plausible, this and other interpretive models can not be presently decisively tested owing to the bright central nucleus and the limited spatial resolution and dynamic range of existing observing methods. The same limitations render any subtle details close to the nucleus indiscernable in our images.

Nonetheless, there are strongly suggestive hints of an O III counterpart to the NE radio jet (Fig. 2). A companion $(\mathrm{H}+\mathrm{N}$ II) counterpart can not be found in the present data, but the existence of such a faint counterpart, e.g., one in which the $\mathbf{O}$ III to $(\mathbf{H}+\mathbf{N}$ II) ratio resembles that of the nucleus, can not be ruled out. Conceivably, the nucleus could be the source of heating and ionization throughout the inner 5 " region. However, this conclusion must be viewed cautiously. If the NE radio lobe interacts with (e.g., is confined by) the gas near the nucleus, as one can infer from the radio morphology (Icke 1984), then a multiplicity of heating processes may contribute to the local energy and ionization budget. Such processes seem to play an important role in, e.g., 3C305 (Heckman et al. 1982). (On the other hand, the polarization observed at $\lambda 2 \mathrm{~cm}$ in the NE radio lobe [WU83] may suggest that the lobe lies in front of, rather than imbedded in, the ionized gas seen in the same direction. If true, then the SW lobe may lie symmetrically behind the disk.)

The bright NE ridge of $O$ III emission which crosses the nucleus in PA $\sim 30^{\circ}$ has an O III flux comparable to the nucleus itself. This ridge causes optical observations obtained through different apertures and/or seeing conditions to be disparate. (Such disparities have been noted in the past literature). More optimistically, there is a high likelihood that emission-line images obtained with subarcsecond resolution will reveal a wealth of new information about how matter flows into or out of an active nucleus. Speckle observations by Meaburn et al. (1982) support this possibility, albeit tentatively. Future subarcsecond studies could reveal the structural counterparts to the kinematic clouds reported by Walker (1968) and others.

\section{b) The Tongue}

Just beyond the tip of the radio jet $\sim 5^{\prime \prime} \mathrm{NE}$ of the nucleus, Figs. 1 and 2 reveal a relatively bright extension of $O$ III emission. We designate this feature "the tongue". Note that the radio map of WU83 implies a sharp emission cutoff all around the NE radio lobe including the head of the jet. Thus the existence of the $\mathrm{O}$ III tongue beyond the radio jet may imply that the collimated energy "beam" responsible for creating the radio lobe actually penetrates beyond the NE radio lobe. Alternatively, the medium which lies beyond the radio lobe may be ionized by an agent generated along the outer lobe boundary. The "plume" described later adds some credence to this speculation.

N84 and BH79 have made limited measurements of emission-line ratios near the tongue. These observations imply, but do not prove, that the tongue is ionized in the same way as the nucleus. More detailed observations from the ground are readily possible, but unfortunately not available in the literature. Small apertures $\left(\sim 2^{\prime \prime}\right)$ and a careful correction for stellar absorption lines are required for meaningful results in future measurements.

\section{c) The Bib}

Like the tongue, the bib is more prominent in $\mathrm{O}$ III than in $(\mathrm{H}+\mathrm{N}$ II), and so both features may share a common ionization agent. However, the bib is found (only) in the SE quadrant in which no bright radio emission is detected. Our longslit HGVS data confirm the existence of the bib, and demonstrate that it is more highly ionized and/or hotter than the $\mathrm{H} \alpha$ crescent material described below [O III] $\lambda 5007$ is $\sim 5$ to 10 times stronger relative to both [O II] $\lambda 3727$ and $\mathbf{H} \beta$ in the bib than it is in the $\mathrm{H} \alpha$ crescent). The most likely ionization and heating agents are photons and/or a particle wind, presumably coming directly from the nucleus.

Nuclear ionization should be emitted symmetrically. Since the bib is found only in one quadrant, a nuclear ionization model requires that the disk interior to the bib is more 
opaque in the other quadrants than to the SE. Note the existence of a gap to the $\mathrm{SE}$ in the crescent of $\mathrm{H}$ II regions, called the "spiral" $\mathrm{H} \alpha$ nebula in Fig. 1. The spiral nebula, if it is an effective absorber of the ionizing agent from the nucleus, could explain why the bib is fully developed only to the SE (see below).

Both Walker (1968) and A83 measured the O III profile shapes near the bib. Walker's data, summarized in Fig. 9 of SYL83, show that velocities change erratically by $\geqslant 50$ $\mathrm{kms}^{-1}$ (relative to nearby gas) in the bib.

\section{d) The Ha Crescent or Spiral Nebula}

The $(\mathrm{H}+\mathrm{N}$ II) image in Fig. 1 shows a bright crescent or partial spiral feature emanating clockwise from the region $\sim 5$ " $\mathrm{S}$ of the nucleus. Some evidence of the same structure was also seen by Bertola (1966). Along this feature, the surface brightness appears to be lumpy. N84 and SBB each examined different lumps and concluded that they are $\mathrm{H}$ II regions (e.g., material ionized by OB stars). Our HGVS data provide further observational support for the idea, since the spectra of the $\mathrm{H} \alpha$ crescent regions resemble the spectra of metal-rich $\mathrm{H}$ II regions in other galaxies. Thus we shall assume that all lumps are discrete $\mathrm{H}$ II regions imbedded in an ionized substrate. Organized collections of nebulae this bright are not generally seen in other Seyfert galaxies [from our unpublished $(\mathrm{H}+\mathrm{N}$ II) and $\mathrm{O}$ III images]. On the other hand, examples of very similar morphological features are found in NGC 3310 (Balick and Heckman 1981; Heckman and Balick 1980; and other references therein), and NGC 1097 (Wolstencroft, Tully, and Perley 1984). Both NGC 1097 and NGC 3310 are highly unusual disk galaxies with optical emission "jets" (tidal debris?) extending beyond their disks.

Curiously, there is only a vague correlation between the surface brightness of $(\mathbf{H}+\mathrm{N}$ II) and the blue stellar continuum, respectively. The absence of a strong correlation was not noticed by Bertola (1966) and Alloin et al. (1981) since the continuum was not separated from the line emission. Given the very strong (stellar?) P Cygni profile shapes in the UV spectra of some of the nebulae (SBB82), rather unusual internal sources of ionization and heating can be expected in some or all of the $\mathrm{H}$ II regions within the spiral. Indeed, some of the $\mathrm{H}$ II regions do not appear to have associated optical continuum regions (see Fig. 1). Kinematic and spectroscopic studies, many of which are feasible from the ground, can be undertaken in order to understand better the nature and history of this active region of star formation and its possibly strange sources of ionization.

If the $\mathbf{H} \alpha$ crescent is really a spiral, then like the spiral arms of NGC 1068, the spiral is a trailing one (Alloin et al. 1981). Therefore it is unreasonable that the $\mathrm{H} \alpha$ spiral is caused by collimated ejecta moving outward from the nucleus and being swept by the ram pressure of the rotating disk material (such material would have to be streaming against the momentum of the rotating disk). Balick and Heckman (1981) suggested that the $(\mathrm{H}+\mathrm{N}$ II) and $\mathrm{O}$ III crescent in NGC 3310 was the phosphorescent wake left by a compact mass as it fell via dynamical friction towards the nucleus through the rotating gas of the disk. Such a suggestion may apply to the $\mathrm{H} \alpha$ spiral in NGC 1068 . However, the scenario above has only circumstantial support.

Finally, we note that NGC 1068 is a strong source of extended molecular (e.g., SYL83) and infrared (TBWH, TH, Hildebrand et al. 1977) emission. Although this molecular and dust emission has not been conclusively proven to be associated with the crescent, the available evidence certainly points toward a close association: see SYL83 and TBWH for a detailed discussion. In particular, TBWH found that the strongest extranuclear $10-\mu$ source lay $6 " \mathrm{~S}$ and $12^{\prime \prime} \mathrm{W}$ of the nucleus. This coincides nicely with the position of the brightest $\mathrm{H}$ II region in the $\mathrm{H} \alpha$ crescent, providing additional support for intense local star formation there. (Molecular and far-IR emission are often associated with regions of active star formation.) Further observations to clarify the distribution of emission of the molecular regions (which contain the vast majority of dust mass-TH) would be of interest. If the cold clouds are local to the crescent, as SYL83 and TBWH argue, then large and nonuniform extinction may be present. In this regard, it is noteworthy that SYL83 and TBWH suggest that like the $\mathrm{H} \alpha$ crescent, the surface brightness of $\mathrm{CO}$ and $10-\mu$ emission is smallest in the SE quadrant (where the bib is found).

\section{e) The Plume}

Lying about 20" ENE of the nucleus is the base of a plume- or fan-shaped feature. The plume neither points toward nor away from the nucleus. Rather, it projects onto a gap between spiral arms, and its base lies between two bright $H$ II regions (one in an arm and the other in the $\mathrm{H} \alpha$ crescent). The plume is seen only in $\mathrm{O}$ III (Figs. 1 and 2), and there is no evidence of a link between the plume and the nucleus at any wavelength. Our long-slit data reveal the plume material to be highly ionized and of high excitation ([O III $] \lambda \quad 5007 \geqslant 5$ times $\mathrm{H} \beta$; [ $\mathrm{Ne} \mathrm{v}] \lambda 3426$ easily detectable). The plume material then resembles the nuclear (inner 5 ") region in its ionization properties. A small O III kinematic anomaly is also associated with the plume (see SYL83, Fig. 9).

Among the possible explanations for the plume which can be readily tested from the ground in conditions of mediocre seeing are the following:

(1). the bib, tongue, and plume share a common ionization source and similar internal kinematics;

(2). the plume is part of a larger feature lying on the far side of the disk of NGC 1068 and visible only between two spiral arms (note: 1 and 2 are compatible); and

(3). the plume derives its ionization and heating externally, probably from the adjacent $\mathrm{H}$ II regions and/or the nucleus.

\section{f) The NE and SW H II Complexes}

Our imaging and long-slit spectroscopic data suggest that superficially, at least, these complexes resemble the giant extragalactic $\mathbf{H}$ II regions seen in other late-type galaxies such as M101. The complexes are found on opposite sides of the nucleus along the major axis, but this may be fortuitous. The spectral character of the NE complex reported by N84 shows indications of ionization and heating by both normal stars and shocks. Although this may be unusual, the strong ultraviolet $\mathbf{P}$ Cygni profiles reported by SBB elsewhere in NGC 1068 argue that strong stellar winds are certainly a plausible explanation for the unusual line ratios seen by N84.

\section{g) Possible Supernovae (not identified in Fig. 1)}

The $(\mathbf{H}+\mathbf{N}$ II) frame shows several spatially unresolved emission knots, none of which show bright continuum counterparts. The two brightest knots lie at (angular offset, PA) $\left(30^{\prime \prime}, 330^{\circ}\right),\left(40^{\prime \prime}, 220^{\circ}\right)$ with respect to the nucleus. The latter knot lies close to the SW H II complex. By analogy, e.g., to 
SNR NGC 4449 (Balick and Heckman 1978), these objects could be young supernovae. Our HGVS spectrum of the SW knot and surroundings is characterized by strong $\mathrm{H} \beta$ and weak [O III] $\lambda 5007$ and [O II] $\lambda 3727$ emission. It resembles the knots in the $\mathrm{H} \alpha$ crescent spectroscopically, and may simply be a small $\mathrm{H}$ II region of unusually high brightness. Further spectrophotometric observations are warranted. Neither object has been mentioned by WU82 and WU83 as a probable small radio source.

\section{CONCLUSION}

NGC 1068 is a nearby gas-rich galaxy in which a highly visible and extremely active nucleus is imbedded. As such, it affords a potentially excellent observational opportunity to look for clues of a strong interaction between an active nucleus and its surroundings. Such footprints are indeed detectable. However, there are many different kinds of footprints, and they do not yet provide decisive, unambiguous answers regarding the manner in which nuclear activity may be spawned and nurtured by the near-nuclear environment.

On close inspection, many of the footprints in the disk of NGC 1068 point away from the nucleus; the radio jets, the tongue and bib, and probably also the plume, may well be a result of nuclear outflow rather than a hint of the origin of nuclear activity. On the other hand, there is a bright crescent of $\mathrm{H} \alpha$ emission which encircles the nucleus in three quadrants. Very vigorous star formation is in progress in the crescent. The crescent could be interpreted as a snowplow driven by a nuclear wind or explosion into the galaxy's gas-rich environment. However, no other Seyfert galaxy clearly shows a similar morphological feature. On the other hand, there are several gas-rich and somewhat disturbed galaxies, such as NGC 1097 and NGC 3310, which do have similar crescents. The nuclei of these galaxies are not Seyfert nuclei although nuclear emission lines have been detected. Intriguingly, such galaxies may be pre- or post-eruptive Seyfert galaxies.

NGC 1068 is sometimes characterized as a prototype Seyfert galaxy (e.g., WU82) in spite of its higher than usual bolometric nuclear luminosity. From the perspective of our imaging survey, the galaxy exhibits the richest display of emission-line features of any nearby Seyfert galaxy yet observed, including others of approximately the same Hubble type and/or distance (e.g., NGC 3227, NGC 4051, NGC 4151, NGC 4235). Rather than being a prototype Seyfert galaxy, we propose that NGC 1068 be considered a propitiously nearby example of an uncharacteristically gas-rich Seyfert host whose nucleus is atypically luminous.

Our data, taken together with available ultraviolet, infrared, and $\mathrm{mm}$-wave $(\mathrm{CO})$ data demonstrate that in addition to its active nucleus, NGC 1068 is also hosting an exceptionally luminous starburst in its near-nuclear zone (see TBWH and Weedman and Huenemoerder 1984). It will clearly be of great interest to determine whether a causal link between nuclear starbursts and "true" nuclear activity exists. Detailed study of NGC 1068 and a statistical investigation of a large sample of active galaxies are both needed to test this possibility.

The variety of phenomena in NGC 1068 can be compared to the other nearby luminous active galaxies which have been carefully studied, such as Centaurus $\mathbf{A}$ (reviewed recently by Ebneter and Balick 1983). Unfortunately, Cen A does not reveal its nucleus or NZ to the optical observer because of its thick edge-on disk. Yet, very close to this disk are some interesting optical features. Relevant here is a possibly shock-ionized and smooth component studied by Phillips (1981) and Phillips et al. (1984), seen above and below the disk. Superficially, this feature may be related to the plume in NGC 1068.

While the NZ in the nearby Seyfert galaxy NGC 4151 bears some similarity to that in NGC 1068 (e.g., Ulrich 1973; Heckman and Balick 1983; Wilson and Ulvestad 1983), it is apparently lacking the bright giant $\mathrm{H}$ II regions and copious molecular gas found in NGC 1068. The bright [O III] plume or jet found in NGC 4151 may be the counterpart of the plume in NGC 1068, although the latter is not nearly so radially oriented ${ }^{\dagger}$.

In spite of the plethoric observations of the NZ in NGC 1068 , including those reported here, no coherent model for this interesting region has really emerged. Indeed, it isn't entirely clear that all of the near-nuclear phenomena derive directly or even indirectly from the luminous active nucleus of the galaxy. Still the many near-nuclear features are bright and near enough that they hold the promise of revealing their nature and relationship to the nucleus before the Space Telescope. Thus the images and discussions in this paper should be considered both as a guide to the future students of this important galaxy as well as a reference for research on other hybrid starburst/Seyfert galaxies.

We wish to thank the staff of Kitt Peak National Observatory, especially Jeanette Barnes, Bill Ditsler, Jim De Veny, and Harvey Butcher, for their devotion to and patience with guest observers in general and us in particular. B. B. and T. M. H. further appreciate financial support from the National Science Foundation for Grants Nos. AST-82-08041 and AST-82-16553, respectively. The generous hospitality of Leiden Observatory to B. B. was critical for the completion of the data analysis. T. M. H. is grateful for support from the Alfred P. Sloan Foundation and from the NASA Goddard Space Flight Center's Laboratory for Astronomy and Solar Physics (NASA/ASEE Summer Faculty Fellowship).

\footnotetext{
${ }^{+}$Cen A and NGC 4151 have turned out to be interesting and highly structured x-ray sources. Unfortunately, comparable x-ray data for NGC 1068 do not seem to be available.
}

\section{REFERENCES}

\footnotetext{
Alloin, D., Lagues, P., Pelat, D., and Despiau, R. (1981). Astron. Astrophys. Suppl. 43, 231.

Alloin, D., Pelat, D., Boksenberg, A., and Sargent, W. L. W. (1983). Astrophys. J. 275, 493.

Balick, B., and Heckman, T. M. (1978). Astrophys. J. Lett. 226, L7.

Balick, B., and Heckman, T. M. (1979). Astron. J. 84, 302.

Balick, B., and Heckman, T. M. (1981). Astron. Astrophys. 96, 271.
}

Balick, B., and Heckman, T. M. (1982). Annu. Rev. Astron. Astrophys. 20, 431.

Bertola, F. (1966). Publ. Obbs. Padova No. 34.

Burbidge, E. M., Burbidge, G. R., and Pendergast, K. H. (1959). Astrophys. J. 130, 26.

Ebneter, K., and Balick, B. (1983). Publ. Astron. Soc. Pac. 95, 675.

Heckman, T. M., and Balick, B. (1979). Astron. Astrophys. 76, L7. 
Heckman, T. M., and Balick, B. (1980). Astron. Astrophys. 83, 100.

Heckman, T. M., and Balick, B. (1983). Astrophys. J. 268, 102.

Heckman, T. M., Miley, G. K., Balick, B., Van Breugel, W. J. M., and Butcher, H. R. (1982). Astrophys. J. 262, 529.

Hildebrand, R. H., Whitcomb, S. E., Winston, R., Steining, R. F., Harper, D. A., and Moseley, S. H. (1977). Astrophys. J. 216, 698.

Icke, V. (1984). Submitted to Nature.

Jones, T. W., Leung, L. M., Gould, R. J., and Stein, W. A. (1977). Astrophys. J. 212, 52.

Meaburn, J., Morgan, B. L., Vine, H., Pedlar, A., and Spencer, R. (1982). Nature 296, 331.

Miller, J. S., and Antonucci, R. J. (1983). Astrophys. J. Lett. 271, L7.

Nishimura, M., Kaneko, N., and Toyama, K. (1984). Astron. Astrophys. 130, 46.

Pedlar, A., Booker, R. V., Spencer, R. E., and Stewart, O. J. (1983). Mon. Not. R. Astron. Soc. 202, 647.

Pelat, D., and Alloin, D. (1983). Astron. Astrophys. 81, 172

Phillips, M. M. (1981). Mon. Not. R. Astron. Soc. 197, 659.

Phillips, M. M., Taylor, K., Atherton, P. D., and Axon, D. J. (1984). Preprint.
Sanders, R. H., and Bania, T. M. (1976). Astrophys. J. 204, 341.

Scoville, N. Z., Young, J. S., and Lucy, L. B. (1983). Astrophys. J. 270, 443.

Snijders, M. A. J., Briggs, S. A., and Boksenberg, A. (1982), Proceedings of the Third European IUE Conference (European Space Agency, Noordwijk, The Netherlands).

Telesco, C. M., and Harper, D. A. (1980). Astrophys. J. 235, 392.

Telesco, C. M., Becklin, E. E., Wynn-Williams, C. G., and Harper, D. A. (1984). Astrophys. J. 282, 427.

Ulrich, M. H. (1973). Astrophys. J. 181, 51.

Van der Hulst, J. M., Hummel, E., and Dickey, J. M. (1982). Astrophys. J. Lett. 261, L59.

Walker, M. F. (1968). Astrophys. J. 151, 71.

Weedman, D. W., Feldman, F. R., Balzano, V. A., Ramsey, L. W., Sramek, R. A., and Wu, C.-C. (1981). Astrophys. J. 248, 105.

Weedman, D. W., and Huenemoerder, D. P. (1984). Bull. Am. Astron. Soc. $16,440$.

Wilson, A. S., and Ulvestad, J. S. (1982). Astrophys. J. 263, 576.

Wilson, A. S., and Ulvestad, J. S. (1983). Astrophys. J. 275, 8.

Wolstencroft, R. D., Tully, R. B., and Perley, R. A. (1984). Mon. Not. R. Astron. Soc. 207, 889. 